\title{
New Insight into Microvascular Perfusion by Analyzing Pipe Flow in a Cylindrical Elastic Shell
}

\author{
Yanchun $\mathrm{Hu}^{1}$, Anming $\mathrm{Hu}^{2}$, Shenju Song ${ }^{3}$, Yanxia $\mathrm{Hu}^{4}$ \\ ${ }^{1}$ Department of Orthopaedics, The Fifth People's Hospital, Jinan, China; ${ }^{2}$ Shandong Experimental High School, \\ Jinan, China; ${ }^{3}$ Department of Nursing, The Fifth People's Hospital, Jinan, China; ${ }^{4}$ Department of Infectious Diseases, \\ Center for Disease Control and Prevention, Jinan, China
}

Correspondence to: Yanchun Hu, lixing777521@163.com

Keywords: Microvascular, Perfusion, Hypertension, Cylindrical Elastic Shell, Fluid Flow

Received: August 9, $2018 \quad$ Accepted: September 11, $2018 \quad$ Published: September 14, 2018

Copyright $\odot 2018$ by authors and Scientific Research Publishing Inc.

This work is licensed under the Creative Commons Attribution International License (CC BY 4.0).

http://creativecommons.org/licenses/by/4.0/

\section{(c) (i) Open Access}

\section{ABSTRACT}

Background: Microvascular perfusion, a kind of regional perfusion, plays important roles in delivering oxygen and nutrients, and regulating blood pressure and responses to inflammation. Aim: The aim of this research is to analyze the characteristics of microvascular perfusion by conducting pipe flow in a circular elastic tube. Methods: A model was established with circular elastic tube to mimic microvascular perfusion. The velocity of pressure waves was calculated according to the time that the liquid took to spilt over. What's more, the characteristics and significance of microvascular flow and arteriovenous anastomoses (AVAs) were analyzed. Results: It took the liquid about 0.1 second to spill over from the model, and the velocity of pressure waves is greater than $100 \mathrm{~m} / \mathrm{s}$ in the elastic pipe. A mechanical switch structure and the corresponding mechanism were proposed for microvascular perfusion in AVAs. Conclusion: Microvascular perfusion maintains a considerable constancy of hemodynamics in different tissues, when ventricular contraction changes perfusion pressure to meet metabolic demands appropriately. This theory will help us to gain a new perspective in microvascular flow.

\section{INTRODUCTION}

Cardiovascular function and tissue perfusion are strongly affected by microvascular blood flow resistance, which in microcirculation is governed by flow behavior of blood through a complex network of vessels [1]. The main function of the microvascular is to transport materials. Water and solutes are carried by blood through the microvessels and exchanged, through vessel walls, with the surrounding tissues. This transporting function is highly dependent on the architecture of the microvasculature and on the biophysical behavior of blood flowing through it. The microvasculature of a perfused tissue provides a large area 
for exchange of materials, and brings blood into close proximity with each point in the tissue.

Pipe flow is a type of liquid flow within a closed conduit, and it belongs to hydraulics and fluid mechanics. Due to the existence of friction, pipe flow should be driven by a pressure difference. Pascal's law states that the pressure exerted anywhere in a confined incompressible fluid is transmitted equally in all directions throughout the fluid. When fluids are in motion, Pascal's principle does not necessarily apply. Pascal's law, applied in fluid mixtures, is a meanfield theory of amphiphilic action, and interface wetting in glassy liquids, which allows fluids to transmit force through the media like pipes or tubes. For instance, a force applied to a fluid in a pipe can be transmitted to the other end of the pipe via the fluid [2].

It is known that an elastic cylindrical lossless shell immersed in an acoustic fluid can guide acoustic energy longitudinally without attenuation. In fact, for the physical and geometric parameters within suitable ranges, different surface wave modes, with different angular dependence around the cylinder, can be supported by the structure. These modes propagate longitudinally with subsonic phase velocities, and are evanescent in the transverse direction.

For a rigid circular pipe, if it is relatively longer, the value added in the entire pipeline will need some time to build. The value of fluid pressure is the transfer of intermolecular force between molecules, the intermolecular forces gradually spread to the distance, to the end of the pipe. It needs some time for the stress to be fully established. The added value of the pressure in the rigid perfect tube can be regarded as the low frequency pressure wave. Its transmission speed is equal to the speed of sound [3]. To understand the dynamics of blood is a crucial element to discover biological mechanisms, to fully understand hypertension, and to design sophisticated microfluidic devices, for diagnostics.

\section{MATERIALS AND METHODS}

For this research, materials were used as follows: elastic circular tube, rubber tube, connecting pipes, switch, cylindrical containers, a stopwatch, needle electrode, and invasive blood pressure monitor [4]. Tubes with the same external diameter $(6 \mathrm{~mm})$ and inner diameter $(4 \mathrm{~mm})$ were used. The studied samples are made of rubber. Also, an invasive blood pressure monitor was placed in the A side of the tube. The schematic diagram of the equipment is shown in Figure 1.

The elastic circular tube was adopted to take the rubber tube $(\mathrm{AD}$, internal diameter $4 \mathrm{~mm} \times$ external diameter $6 \mathrm{~mm}$, AD was 20.2 meters long). The rubber tube BC as shown in Figure 1 was horizontal placed, which was 20 meters long. $\mathrm{AB}$ was perpendicular to the ground, and was $0.1 \mathrm{~m}$ high equal to $\mathrm{CD}$, which was also perpendicular to the ground. Thus a communicating vessel was formed. The end A was connected with a water container $\mathrm{M}$, and the water height was $0.3 \mathrm{~m}$. There was a switch at the entrance $\mathrm{A}$, and it was shut. A, D were remained at the same level. So, if it was switched off, there would be $0.1 \mathrm{~m}$ high water pressure inside horizontal pipe. After it was switched on, the time that the fluid took to overflow from $\mathrm{D}$ was recorded. In order to facilitate the observation, elastic pipe to take back, so that both ends were near to each other. And all the tubes were fixed on a rigid plate tightly as shown in Figure 2. A invasive blood pressure monitor was placed in the A side of the tube. The rise of the A end pressure was the

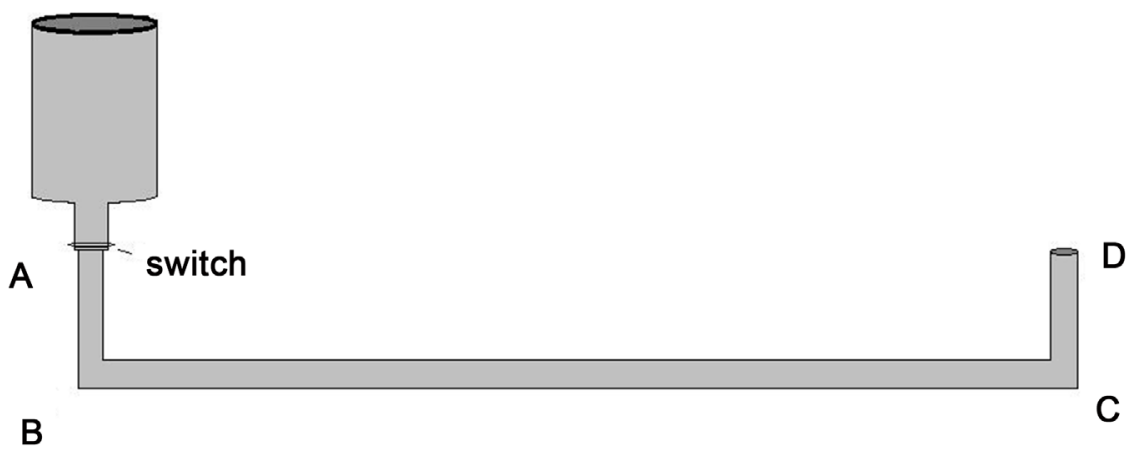

Figure 1. Schematic diagram of the equipment. 


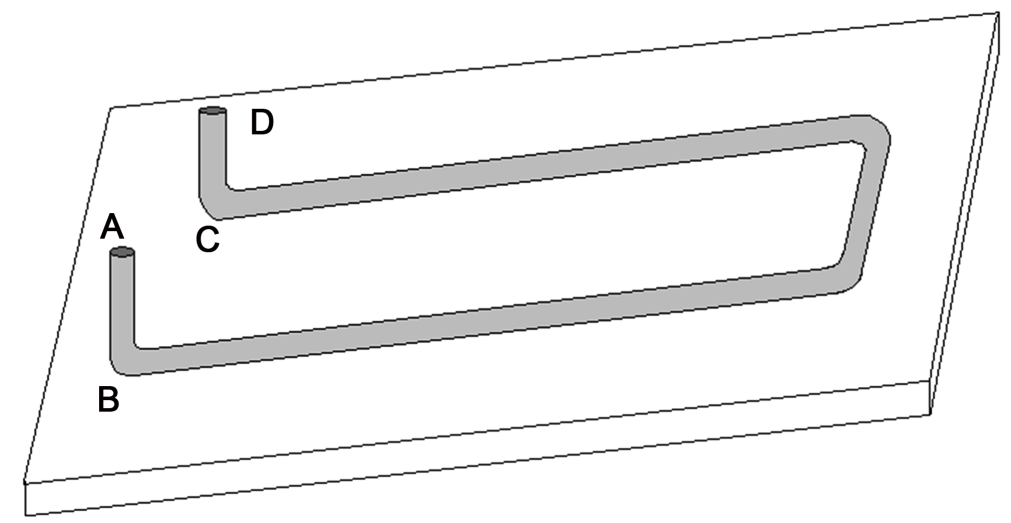

Figure 2. Geometry of the model.

sign for the A end switch to turn on. Place a needle electrode right out of the $\mathrm{D}$ end. When the water is flowing out of the $\mathrm{D}$ end, the needle electrode will be soaked in the water, current changes were produced. The change of electric current was the sign of water outflow. The pressure detector and the needle electrode recorder have synchronous clock, and the difference of the clock is the pressure transmission time. It takes about 0.1 seconds from the opening of $A$ side switch to the $\mathrm{D}$ end start to flow out water.

\section{RESULTS AND DISCUSSION}

A straight rigid circular pipe filled with water and pistons were placed at each end, as a consequence, pressure exerted against the left piston would be transmitted throughout the liquid and against the right piston as in Figure 3. If the pipe was $1000 \mathrm{~m}$ long, the left piston began to move about $1 \mathrm{sec}$ later after the right side of the applied stress. Notably, the pistons are simply "plugs" that can slide freely but snugly inside the tube. When the water is just beginning to flow, the pressure that the left piston exerts against the water will be exactly equal to the pressure the water exerts against the right piston. Or when the water has been flowing, the pressure that the left piston exerts against the water will be greater than the pressure the water exerts against the right piston.

Pressure wave, a type of longitudinal waves (longitudinal waves are waves that have the same direction of vibration as their direction of travel), is formed from alternating compressions and rarefactions. In rigid pipeline, the pressure wave will travel in the medium at a velocity $\mathrm{C}$ equal to the velocity of sound in an infinite expansion of the medium, and given by an equation $(\alpha)$ :

$$
C=\sqrt{K} / \rho
$$

where $K=$ Bulk modulus of liquid, $\rho=$ Mass density of fluid [5] [6]. If the pipe is elastic, the velocity of propagation will be less than that given by the equation $\alpha$. The result we obtained showed that it took about $0.1 \mathrm{~s}$ from the opening of switch at A to starting to flow out water at end D.

As shown in the above experiments, because $\mathrm{D}$ is open to the air, the entire pipeline cannot form the same hydrostatic pressure as the A. When D end starts to flow out water, the stress field is not fully established in the whole pipeline. For elastic pipe, flow out water from $\mathrm{D}$ end means the pressure starts to rise at the end of pipe. This is sufficient to change the balance of the end pressure to make the water flow out of the water. The pressure transmission speed is quite fast. In a very short time to produce pressure difference in tube end that pushes the liquid to move forward.

In the human body, the arterial blood pressure pulse is one of the most fundamental physical quantities related to haemodynamics. Measurement techniques have been developed, which at times have obscured the true nature of this pulsatile phenomenon. Blood pressure is, however, a composite wave that undergoes significant changes in its contour while traveling within the arterial system. Thus, the term "blood pressure", when used alone, does not express this wave nature. Pulse waves, which are usually 


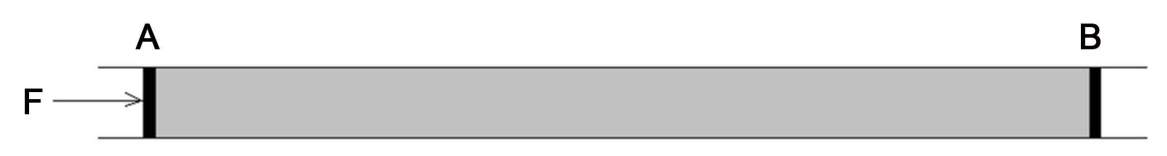

Figure 3. Schematic diagram of pipe with pistons.

measured, are waves that are oscillating perpendicularly to the direction of propagation. The pulse waves caused from LV (left ventricular) ejection that increase the arterial wall linearly [7]. Pulse wave velocity (PWV) is obviously lower than the speed of sound, for a health younger, pulse wave velocity is less than 20 $\mathrm{m} / \mathrm{s}$ in arterial [8].

In order to detect pulsation changes in blood volume in the microvascular tissue bed, the photoplethysmography (PPG) is often used [9]. Because of the ventricular contraction, a volume of blood is squeezed into the periphery. Then microvascular perfusion get to peak that can be recorded by PPG. Combined with simultaneous cardiac ultrasound, Mitral valve closure can be measured as a starting point for increased ventricular pressure. PPG peak can be taken as a sign of the peak value of microcirculation perfusion, it appears only 0.2 seconds after aortic valve opens. This makes tissue perfusion almost at the same time, not because of the distance from the heart is different. Meanwhile, peak of PPG was earlier than pulse wave get to the periphery.

The capillary net is a strongly bifurcate network of small vessels, and the number of vessels in the capillary system is significantly larger than in the arterial and venous system [10]. As a consequence, the total cross section in the capillary system is about 1000 times larger than in the arterial and the venous system. When the blood flows out of the capillaries into the venules, the total cross-sectional area will gradually become smaller. In this way, the capillary network has formed the pipeline structure which has an enlarged total cross-sectional area in the midsection and lesser total cross-sectional area at each end. When the blood flows through the lumen of a sudden enlargement of the area of the cross section, the blood flow in the diffuser is generated. Due to constant flow through the expansion of the lumen, it will inevitably lead to a reduction in the blood flow velocity. Blood flow through the cross-sectional area with a sudden decrease, will produce a convergence of the flow cross-section, such as the cone shape pipeline blood flow velocity distribution.

Due to small elastic modulus and larger cross-sectional area, the capillaries can behave as a "windkessel", which can cushion the pulsations generated by the heart. When the ventricle contracts, the pipeline of capillary net expands, and the highest value of capillary pressure in capillary network was lower than in the arterioles. According to basic flow equation, it cannot be concluded that capillary network flow resistance is less than the arterioles. The basic flow equation $(Q=\Delta P / R$, blood flow $=$ pressure/resistance) summarizes the relationship between the pressure difference, blood flow, and vascular resistance. The basic flow equation is originated from Ohm's law, based on a fundamental law of physics, which states that the current $(I)$ equals the voltage difference $(\Delta V)$ divided by the resistance $(R)$. In electrical circuits, Ohm's law is applied in the field of mathematics. However, the water analogy can create misconceptions, and awareness that electricity and water behave very differently is important [10].

Loss of mechanical energy of unit mass fluid in the process of movement is called head loss. The kinetic energy of the blood flow generated by the contraction of the heart is ultimately converted into heat because of friction. Order of magnitude, red blood cells with diameters of $7 \mu \mathrm{m}$ must deform to pass through them. Head loss will be large in capillary network [11].

Capillaries are composed of a single layer of endothelial cells which lack of vascular smooth muscle. There is no neural or local metabolic control of capillary vessels, whereas arterioles have much thicker walls with smooth muscles. If we believe the resistance is highest in the capillaries, then why relaxation of vascular smooth muscles should lower blood pressure must be answered.

Mostly, blood develops to become the arterioles in two ways. The first way is to follow a metarteriole forms a specific part of the tissue and through the capillary system. The second way is to enter an arteriovenous anastomosis (AVA) that shortcuts the arterioles and the venules to bypass the tissue [12]. Arteri- 
ovenous anastomoses (AVAs) are low-resistance vessels. They have a relatively larger diameter than capillaries, and they form low-resistance channels by which the high-capacity venous bed can be rapidly perfused. The structure of the blood vessel wall has different elastic modulus. A sphincter-like muscle was formed in the arterioles. It can adjust blood flow into controlled arteriovenous anastomoses. Histologically, AVAs are characterized by an irregular lumina (3 - 8 times larger than those of capillaries) and muscular walls. In cross-sections, the thick walls of the arteriovenous anastomoses consist of circular and longitudinally arranged smooth muscle fibers, the opening is usually narrow and has a stellate outline due to the ridges of smooth muscle cells that protrude into the lumen. There is a notable thickening of the vascular smooth muscle at the origin of AVAs from the parent arterioles.

The thickening tissue of the vascular smooth muscle in the parent arterioles makes it possible to form a lip-type structure. The parent artery, the vascular smooth muscle differs from the endothelial cells in the elastic modulus. The vascular wall tension of the parent artery varies with the intravascular pressure over the cardiac cycle. Therefore, it is structurally possible for the AVAs to close during systole (blood pressure $\geq 90 \mathrm{mmHg}$ ) and to open during diastole (blood pressure $<90 \mathrm{mmHg}$ ) due to higher and lower wall tension in the arterioles, respectively [13].

In fact, if the systolic blood pressure becomes reduced $(<90 \mathrm{mmHg})$ due to bleeding during shock, the arteriovenous anastomoses will open. This phenomenon is characteristic of changes in the microcirculation during shock [14].

Figure 4 showed a tension switch model with different elastic modulus materials. The two valves are symmetrical, as the valve channel between cd and c'd'. a, a' is the fixed axis, triangular acd and a'c'd' are valves and could rotate around a, a' fixed axis. The lines bc and b'c' were rubber rope (mimic endothelial cells), bd and b'd' were cotton rope (mimic vascular smooth muscle). Cotton ropes were longer rubber ropes. The elastic modulus of cotton rope is larger than rubber rope. There is a certain elastic F which generates pressure $<90 \mathrm{mmHg}$. The length: $\mathrm{ac}=\mathrm{ad}, \mathrm{bc}=\mathrm{bd}$. The stress of cotton rope is also $\mathrm{F}$. The $\mathrm{cd}$ and c'd' parallel channel in the open state. The $b$ and b' points in the opposite direction were respectively applied with forces $\mathrm{F}$ and $\mathrm{F}^{\prime}$, respectively, which could generate $\mathrm{a} \geq 90-\mathrm{mmHg}$ pressure intensity in each direction. Due to the elastic modulus of cotton rope was larger than that of rubber rope, under the action of the tension, the valve acd would show a clockwise rotation, while a'c'd' would show a counter-clockwise rotation, resulting in a closed channel. When $\mathrm{F}$ and $\mathrm{F}^{\prime}$ decreased, the channel was opened up with the counter-clockwise rotation of acd and clockwise rotation of a'c'd' [15].

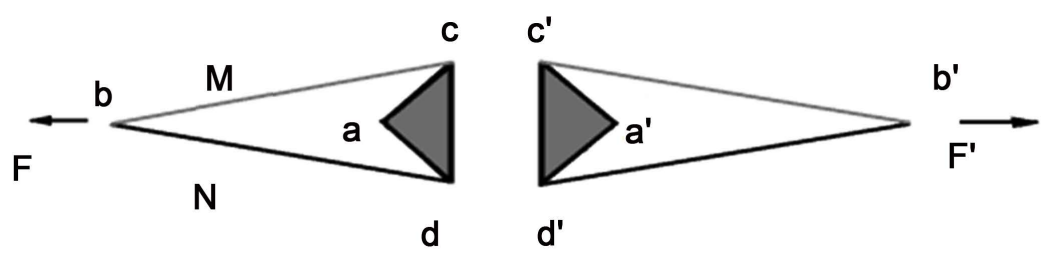

1

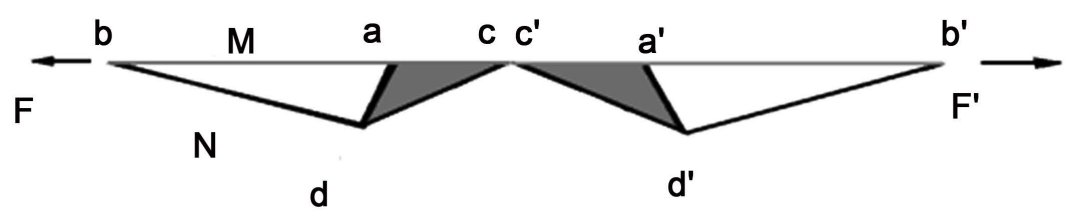

2

Figure 4. The tension switch model for arteriovenous anastomoses. 
Based on the previous studies we conducted, vascular endothelial dysfunction represents an initial step toward hypertension. The endothelium plays multiple pathological and physiological roles, including the regulation of smooth muscle tone. Vascular smooth muscle relaxation or contraction may result in wall tension in arterioles, which also change arteriovenous anastomoses [16].

A number of unresolved issues still remain, particularly with regard to the relationship between blood flow properties as observed in vitro and the functional behavior of blood in the living microcirculation. Future studies should address these issues, including the causes of the discrepancy between the apparent viscosity of blood in microvessels in vivo and that in glass tubes. The in vivo significance of red cell aggregation and sedimentation in both normal and pathological situations remains to be debated. Although measurements of the bulk viscosity of blood and red cell deformability are frequently correlated with specific clinical conditions, the actual significance of such measures with regard to blood circulation is poorly understood. The mechanics of red cell screening in microvascular bifurcations is not well understood, although this phenomenon is probably very significant in smaller bifurcations. The high level of heterogeneity in microvascular hemodynamics is recognized, but efforts to understand its implications for the mass transport functions of the microcirculation are at a relatively early stage.

In this work, we focused on the pipe flow in shell to gain a new perspective in microvascular flow, and the results demonstrated that microvascular perfusion maintains a considerable constancy of hemodynamics in different tissues from head to toe after ventricular contraction. Although it is difficult to get a specific answer or answers to microvascular hemodynamics, understanding the dynamics of blood cells is still a crucial element to discover biological mechanisms. It was reasonable to believe that changes of arteriovenous anastomoses were the key to the development of hypertension. With the development of modern science, branches of medical science have been increasingly coming up with their theory based on accurate physics and pipe flow analysis will help us fully understand the micro circulation.

\section{CONFLICT OF INTEREST}

We all declare that we have no conflict of interest.

\section{REFERENCES}

1. Katanov, D., Gompper, G. and Fedosov, D.A. (2015) Microvascular Blood Flow Resistance: Role of Red Blood Cell Migration and Dispersion. Microvascular Research, 99, 57-66. https://doi.org/10.1016/j.mvr.2015.02.006

2. Chan, H.Y. and Lubchenko, V. (2015) Pressure in the Landau-Ginzburg Functional: Pascal's Law, Nucleation in Fluid Mixtures, a Meanfield Theory of Amphiphilic Action, and Interface Wetting in Glassy Liquids. The Journal of Chemical Physics, 143, 124502. https://doi.org/10.1063/1.4931177

3. Godinho, L., Tadeu, A. and Branco, F.J.G. (2004) Dynamic Analysis of Submerged Fluid-Filled Pipelines Subjected to a Point Pressure Load. Journal of Sound \& Vibration, 271, 257-277. https://doi.org/10.1016/S0022-460X(03)00752-1

4. Drawz, P.E., Abdalla, M. and Rahman, M. (2012) Blood Pressure Measurement: Clinic, Home, Ambulatory, and Beyond. American Journal of Kidney Diseases, 60, 449-462. https://doi.org/10.1053/j.ajkd.2012.01.026

5. Willemet, M. and Alastruey, J. (2015) Arterial Pressure and Flow Wave Analysis Using Time-Domain 1-D Hemodynamics. Annals of Biomedical Engineering, 43, 190-206. https://doi.org/10.1007/s10439-014-1087-4

6. Lin, T.C. and Morgan, G.W. (1956) Wave Propagation through Fluid Contained in a Cylindrical, Elastic Shell. Journal of the Acoustical Society of America, 28, 1165-1176. https://doi.org/10.1121/1.1908583

7. Peltokangas, M., Vehkaoja, A., Verho, J., Huotari, M., Roning, J. and Lekkala, J. (2014) Monitoring Arterial Pulse Waves with Synchronous Body Sensor Network. IEEE Journal of Biomedical and Health Informatics, 18, 1781-1787. https://doi.org/10.1109/JBHI.2014.2328788

8. Nemcsik, J., Cseprekal, O. and Tisler, A. (2017) Measurement of Arterial Stiffness: A Novel Tool of Risk Strati- 
fication in Hypertension. Advances in Experimental Medicine and Biology, 956, 475-488.

9. Allen, J. (2007) Photoplethysmography and Its Application in Clinical Physiological Measurement. Physiological Measurement, 28, R1-39. https://doi.org/10.1088/0967-3334/28/3/R01

10. Huang, T.C., Lin, W.C., Wu, C.C., Zhang, G. and Lin, K.P. (2010) Experimental Estimation of Blood Flow Velocity through Simulation of Intravital Microscopic Imaging in Micro-Vessels by Different Image Processing Methods. Microvascular Research, 80, 477-483. https://doi.org/10.1016/j.mvr.2010.07.007

11. Hu, Y. (2013) Flow Resistance of Vessels with an Enlarged Total Cross-Sectional Area in the Midsection. Open Circulation \& Vascular Journal, 6, 9-12. https://doi.org/10.2174/1877382601306010009

12. Granger, D.N. (1988) Physiology and Pathophysiology of the Microcirculation. American Journal of Otolaryngology, 9, 264-277. https://doi.org/10.1016/S0196-0709(88)80035-8

13. Hutchinson, J. (1986) Association between Stress and Blood Pressure Variation in a Caribbean Population. American Journal of Physical Anthropology, 71, 69-79. https://doi.org/10.1002/ajpa.1330710109

14. Pries, A.R., Secomb, T.W., Gessner, T., Sperandio, M.B., Gross, J.F. and Gaehtgens, P. (1994) Resistance to Blood Flow in Microvessels in Vivo. Circulation Research, 75, 904-915. https://doi.org/10.1161/01.RES.75.5.904

15. Sharma, S., Zhuang, Y., Ying, Z., Wu, A. and Gomez-Pinilla, F. (2009) Dietary Curcumin Supplementation Counteracts Reduction in Levels of Molecules Involved in Energy Homeostasis after Brain Trauma. Neuroscience, 161, 1037-1044. https://doi.org/10.1016/j.neuroscience.2009.04.042

16. Hu, Y. (2015) Photoplethysmographic Waveforms Recorded from Patients with Untreated Hypertension. Clinical Medicine Research, 4, 163-167. https://doi.org/10.11648/j.cmr.20150405.18 